\title{
PRINCIPAL REPRESENTATIONS IN COMMUTATIVE SEMIGROUPS
}

\author{
W. M. PEREL
}

1. Introduction. This paper is intended as a note to [2], and the definitions and notations of that paper will be used throughout, except that $S: T$, where $S$ is a co-ideal and $T$ is the null set will be defined to be $S$, rather than $\mathfrak{M}$. This change will not affect the results of [2]. To avoid repetition, let the terms "semigroup" and "ring" always signify "commutative semigroup" and "commutative ring," respectively. As in [2], let $\mathfrak{M}$ denote a semigroup and $\mathcal{F}$ a family of its co-ideals. Let $S$ and $T$ denote arbitrary elements of $\mathcal{F}$ and let $P$ and $Q$ denote, respectively, prime and primary elements of $\mathcal{F}$. Let $M$ denote a $p$-set contained in $\mathfrak{M}$.

The purpose of [2] was to reproduce, in so far as possible, the ideal theory of rings in semigroups in such a way that ring ideal theory could be obtained as a special case by considering those semigroups which were actually rings. To that end, co-ideals were defined in such a way that the complement of an ideal of a ring, $R$, would be a coideal of the multiplicative semigroup of $R$. In order that those coideals which were not the complements of ring ideals could be discarded when desired, [2] considered only those co-ideals which were members of a particular family, $\mathcal{F}$, of co-ideals of a semi-group, $\mathfrak{M}$, satisfying

I. $S \in F$ implies $S^{\mathbf{\Delta}} \in \mathcal{F}$.

II. The arbitrary union of $\mathcal{F}$-elements is an $F$-element.

III. $S \in \mathcal{F}$ and $M$ a nonempty $p$-set contained in $S$, implies that $S: M \in \mathcal{F}$.

The complements of ring ideals obviously are an example of such a family. If $T$ is any subset of $\mathfrak{M}$, let $C T$ denote the complement of $T$ in $\mathfrak{M}$.

The chief results of [2] were the decompositions of every proper member of a family, $\mathcal{F}$, into a finite union of primary members of $\mathcal{F}$ and into a finite union of proper, pairwise separate, members of $\mathcal{F}$, under certain further restrictions on $\mathcal{F}$. Here another kind of a decomposition will be studied and another ring ideal theory result reproduced in semigroup co-ideal theory.

\section{Preliminary definitions.}

Definition 2.1. If $T, S \in \mathcal{F}, T$ will be called prime to $S$ if there

Received by the editors January 29, 1957. 
exists $a \notin T$ such that $a \cdot S \subset S$.

If $\mathfrak{M}$ is a ring and if $C S$ and $C T$ are ideals, it is seen that $T$ prime to $S$ is equivalent to $C T$ prime to $C S$ in the sense of $[1,16]$, but that this primeness is stronger than that of $[3,25]$.

Obviously $T$ is nonprime to $S$ if and only if $T \supset S: S$.

It will now be necessary to further restrict $\mathcal{F}$ by:

IV. Every nonempty set of elements of $\mathcal{F}$ contains a minimal element.

V. If $a \in S \in \mathcal{F}$, then $S:\{a\} \in \mathcal{F}$.

It should be pointed out that if $\mathfrak{M}$ is a ring and $\mathfrak{F}$ is the collection of the complements of the ring ideals, condition $\mathrm{V}$ is always satisfied and IV is equivalent to the ascending chain condition.

If $S \in \mathcal{F}$, consider the totality of elements of $F$ which contain $S: S$. This collection is nonempty, as it contains $S$, so by IV, there is a $T \in \mathcal{F}$ such that $T$ is minimal nonprime to $S$.

Theorem 2.1. If $T$ is minimal nonprime to $S$, then $T$ is prime.

Proof. If $S: S$ is non-null, $F$ contains a prime element, $P$, such that $T \supset P \supset S: S$, by the first theorem of [2], since $S: S$ is a $p$-set. Since $T$ is minimal, $T=P$.

If $S: S$ is null and $T^{\mathbf{\Delta}}$ is non-null, then $T$ still contains a prime member of $\mathcal{F}$ by the same theorem and this prime member of $\mathcal{F}$ is equal to $T$ by the minimal property. If $T^{\mathbf{\Delta}}$ is null, then $T$ minimal implies $T=T^{\mathbf{\Lambda}}=S: S$ is null, so $T$ is a prime member of $\mathfrak{F}$.

By IV, if $T$ is any co-ideal of $\mathcal{F}$ which is nonprime to $S$, then $T \supset P \supset S: S$, where $P$ is minimal with this property.

Definition 2.2. If $P$ is minimal nonprime to $S$, then $S: P$ is called a principal component of $S$.

N.B. If $P$ is non-null and $S: P$ is non-null, then $S: P$ is an example of a segregated component of [2].

\section{Decomposition theorems.}

THEOREM 3.1. Every element of $\mathcal{F}$ is the union of its principal components.

Proof. If $S \in \mathcal{F}$, let $\left\{P_{\alpha}\right\}$ be all the members of $\mathcal{F}$ which are minimal nonprime to $S$. If any $P_{\alpha} \leftarrow S$, then $S: P_{\alpha}$ is null, so we may suppose that each $P_{\alpha} \subset S$. Since $S$ contains each $S: P_{\alpha}, S \supset \bigcup_{\alpha} S: P_{\alpha}$. Now suppose that $a \in S$. By $V, S: S \subset S:\{a\} \in \mathcal{F}$. Since $S:\{a\}$ is nonprime to $S$, it contains one of the $P_{\alpha}$. But $S:\{a\} \supset P_{\alpha}$ implies that $a \in S: P_{\alpha}$ implies that $a \in \mathrm{U}_{\alpha} S: P_{\alpha}$, so $S=\bigcup_{\alpha} S: P_{\alpha}$.

Definition 3.1. $S=\bigcup_{\alpha} S: P_{\alpha}$ is called the principal representation or principal decomposition of $S$. 
This union may be finite or arbitrary. The principal representation gives less information about $S$ than it does about $\mathcal{F}$. The property of a co-ideal being primary or the union of primary co-ideals is not influenced by increasing the size of $\mathcal{F}$. However, the principal representation may become very much shorter if the size of the family is increased. In fact, if $S: S \in \mathcal{F}$, the principal representation reduces to $S=S:(S: S)$. Conversely, if $P$ minimal nonprime to $S$ exists such that $S=S: P$, then $P=S: S$, so $S: S \in \mathcal{F}$. If $Q$ is primary, then $Q: Q=Q^{\mathbf{\Lambda}} \in \mathcal{F}$, so $Q=Q: Q^{\mathbf{\Lambda}}$ is the principal representation of $Q$.

Restriction $\mathrm{V}$ on the family will now be dropped.

THEOREM 3.2. If $S=\bigcup_{\alpha} Q_{\alpha}$, where the $Q_{\alpha}$ are primary members of $\mathcal{F}$, then $S$ is the union of its principal components. Further, if $U_{\alpha} Q_{\alpha}$ is a finite union, $S$ has only finitely many distinct principal components.

Proof. We may suppose now that for all $\beta, Q_{\beta} \nsubseteq U_{\alpha \neq \beta} Q_{\alpha}$ and that the $Q_{\alpha}^{\mathbf{\Lambda}}$ are distinct. Compare [2, $\left.\$ 4\right]$.

Contention. Each $Q_{\alpha}$ is nonprime to $S$. If $Q_{\beta}$ is prime to $S$, then there exists $a \notin Q_{\beta}$ such that $a Q_{\beta} \subset S$. Now $a Q_{\beta}$ is disjoint from $Q_{\beta}$, so $a Q_{\beta} \subset \bigcup_{\alpha \neq \beta} Q_{\alpha}$, which implies that $Q_{\beta} \subset \bigcup_{\alpha \neq \beta} Q_{\alpha}$, contradicting the above assumption.

So each $Q_{\alpha}$ contains $P_{\alpha}$, where $P_{\alpha}$ is minimal nonprime to $S$. Actually $P_{\alpha} \subset Q_{\alpha}^{\mathbf{\Delta}}$, so since $Q_{\alpha}$ is primary, $P_{\alpha} Q_{\alpha} \subset Q_{\alpha}^{\mathbf{\Delta}} \cdot Q_{\alpha} \subset Q_{\alpha} \subset S$, i.e. $Q_{\alpha} \subset S: P_{\alpha}$. If the $\left\{P_{\gamma}\right\}$ are all of the minimal elements of $F$ containing $S: S$, certainly the $\left\{P_{\alpha}\right\}$ are among them. We have $S=\cup_{\alpha} Q_{\alpha}$ $\subset \bigcup_{\alpha} S: P_{\alpha} \subset U_{\gamma} S: P_{\gamma}$. On the other hand $U_{\gamma} S: P_{\gamma} \subset S$, as before.

Suppose now that $S=Q_{1} \cup \ldots \cup Q_{n}$ is a normed union. See $[2, \S 4]$. Suppose that $P$ is minimal nonprime to $S$. If $P$ is null, then $S: P=S$ is the only principal component and the result is proved. Otherwise the fourth theorem of [2] shows that $S: P$ equals the union of those $Q_{i}$ which contain $P$. In fact, $S: P=S: M$, where $M$ is the intersection of the co-radicals of those $Q_{i}$ which contain $P$. For, again using [2, Theorem 4], $S: M$ is the union of those $Q_{i}$ which contain $M$ and, as each $Q_{i}$ which contains $P$ also contains $M, S: P \subset S: M$. On the other hand, $P \subset Q_{i}$ implies that $P \subset Q_{i}^{\mathbf{\Lambda}}$, so that $P \subset \cap_{Q i \supset P} Q_{i}^{\mathbf{A}}=M$, so $S: P \supset S: M$. Thus the number of possible distinct principal components of $S$ is bounded by the number of such $M$ 's, i.e. by the number of non-null subsets of the $n Q_{i}$, or by $2^{n}-1$.

4. An application. The principal decomposition theory has a particularly interesting application to single-primed families, i.e. those in which every prime element is maximal. In this case [2, Lemma 1$]$ shows that every principal component is a primary element of the 
family. Thus, by Theorem 3.1, every member of a single-primed family, satisfying assumptions I through $\mathrm{V}$, is a union of primary members of the family. For this special case the result is achieved without making assumptions (a) and (b) of $[2, \S 7]$.

\section{BIBLIOGRAPHY}

1. W. Krull, Idealtheorie, Ergebnisse der Mathematik, vol. 4, Part III, Berlin, 1935.

2. R. E. MacKenzie, Commutative semigroups, Duke Math. J. vol. 21 (1954) pp. $471-477$.

3. B. L. van der Waerden, Modern algebra, vol. 2, 2d ed., New York, 1950.

Texas Technological College

\section{A LIMIT THEOREM FOR PROCESSES WITH STATIONARY INDEPENDENT INCREMENTS}

FRANK KOZIN

I. Introduction. Let $[X(t) ; 0 \leqq t \leqq 1]$ be the Wiener process. That is, a Gaussian process of real valued variables with $E[X(t)]=0$ and $E[X(s) X(t)]=\min (s, t)$. Levy [1] showed that with probability one

$$
\lim _{N \rightarrow \infty} \sum_{k=1}^{N}\left[X\left(t_{k}\right)-X\left(t_{k-1}\right)\right]^{2}=1 \quad \text { for } 0=t_{0}<t_{1}<\cdots<t_{N}=1 .
$$

Cameron and Martin [2] showed this independently for $t_{k}=k / 2^{N}$. Baxter [3] recently extended these results by the following theorem:

THEOREM. If $[X(t) ; 0 \leqq t \leqq 1]$ is a Gaussian process satisfying $E[X(t)]=m(t)$ and $E[X(s) X(t)]-m(s) m(t)=r(s, t), r(s, t)$ is continuous in $0 \leqq s, t \leqq 1$ and has uniformly bounded second derivatives for $s \neq t$.

Let

$$
\begin{aligned}
& D^{+}(t)=\lim _{s \rightarrow t^{+}} \frac{r(t, t)-r(s, t)}{t-s}, \\
& D^{-}(t)=\lim _{s \rightarrow t^{-}} \frac{r(t, t)-r(s, t)}{t-s},
\end{aligned}
$$

and

$$
f(t)=D^{-}(t)-D^{+}(t) .
$$

Then with probability one

Received by the editors November 2, 1956. 